\title{
Simulation of Benchmark Cases with the Terminal Area Simulation System (TASS)
}

\author{
Nash'at N. Ahmad*, Fred H. Proctor ${ }^{\dagger}$ \\ NASA Langley Research Center, Hampton, Virginia, 23681
}

\begin{abstract}
The hydrodynamic core of the Terminal Area Simulation System (TASS) is evaluated against different benchmark cases. In the absence of closed form solutions for the equations governing atmospheric flows, the models are usually evaluated against idealized test cases. Over the years, various authors have suggested a suite of these idealized cases which have become standards for testing and evaluating the dynamics and thermodynamics of atmospheric flow models. In this paper, simulations of three such cases are described. In addition, the TASS model is evaluated against a test case that uses an exact solution of the Navier-Stokes equations. The TASS results are compared against previously reported simulations of these benchmark cases in the literature. It is demonstrated that the TASS model is highly accurate, stable and robust.
\end{abstract}

\section{Introduction}

$\mathrm{T}$ he Terminal Area Simulation System (TASS) is a state-of-the-art, cloud-resolving, large eddy simulation atmospheric flow model. TASS computes the primitive variables non-hydrostatic equations in three dimensions and the model is capable of resolving flows at multiple spatial (spatial resolutions varying from less than a meter to $2 \mathrm{~km}$ in the horizontal) and temporal scales (few minutes in case of wake decay simulations to hours for studying convective phenomena). The model also solves prognostic equations for potential temperature, water vapor, cloud droplets, ice crystals, rain, snow and hail. Phase changes between liquid water and ice as well as submodels for cloud and precipitation microphysics are included. Subgrid scale diffusion is parameterized via Smagorinsky-type turbulence closure (Smagorinsky 1963) and surface layer processes are computed based on Monin-Obukhov similarity theory (Stull 1997).

The TASS model has been extensively validated in the past against experimental data and applied to a diverse set of problems ranging from the simulation of shallow cumulus to supercell storms, including convective phenomena such as downbursts, gust fronts and hail storms (Proctor 1987; Proctor and Bowles 1992; Proctor et al. 2002). Although, TASS was initially developed to simulate convective processes, it has been used successfully to simulate the transport and dispersion of hazardous materials from nuclear weapon bursts (Bacon and Sarma 1991). TASS simulations of large urban fires have been used to define source terms for mesoscale models (Bacon et al. 1986). More recently, TASS has been employed to study the decay and transport of wake vortices generated by various aircraft under different conditions of atmospheric stability and turbulence (Proctor 1996; Han et al. 2000a; Han et al. 2000b; Proctor 2009).

In this study the dynamical core of the TASS model is evaluated against benchmark cases and compared with results from other numerical models such as the National Center for Atmospheric Research's (NCAR) state-of-theart Weather Research and Forecast (WRF) model and the Advanced Regional Prediction System (ARPS). A detailed description of the WRF model is given in Klemp et al. (2000) and the ARPS model is described in Xue et al. (2000). Over the years a suite of idealized test cases have been developed for the validation and verification of atmospheric models. Although these cases can evaluate a model mostly in qualitative terms, they nonetheless provide a useful measure to assess a model's capability to simulate the fundamentals of atmospheric hydrodynamics and thermodynamics. Three idealized test cases were used in this study - the non-linear density current simulation (Straka et al. 1993); convection of a warm bubble in a neutral atmosphere (Bryan and Fritsch 2002); and the propagation of non-hydrostatic inertia gravity waves (Skamarock and Klemp 1994). In addition to the idealized test cases mentioned above, the Beltrami flow problem was also computed with TASS and compared against the exact solution (Shapiro 1993).

\footnotetext{
${ }^{*}$ Research Aerospace Engineer, NASA, Hampton, Virginia. Senior Member, AIAA.

${ }^{\dagger}$ Senior Research Scientist, NASA, Hampton, Virginia. Senior Member, AIAA. 


\section{Governing Equations}

The complete governing equations of the TASS model consist of conservation of mass, momentum and energy in three dimensions that include the subgrid-scale diffusion processes and comprehensive cloud and ice microphysics (Proctor 1987). In this study, the dynamical core of the TASS model is evaluated and therefore, the diffusion processes are ignored and the atmosphere is assumed to be dry. The simplified equations can then be written as follows:

$$
\frac{\partial u_{i}}{\partial t}+\frac{H}{\rho_{0}} \frac{\partial p^{\prime}}{\partial x_{i}}=-\frac{\partial u_{i} u_{j}}{\partial x_{j}}+u_{i} \frac{\partial u_{j}}{\partial x_{j}}+g(H-1) \delta_{i 3}
$$

where, $u_{\mathrm{i}}$ and $u_{\mathrm{j}}$ are the velocity components, $x_{\mathrm{i}}$ and $x_{\mathrm{j}}$ are the spatial dimensions, $p$ is the pressure, $g$ is the acceleration due to gravity, and $\delta$ is the Kronecker delta. $H$ is the density ratio defined by:

$$
H \equiv \frac{\rho_{0}}{\rho}=\frac{\theta}{\theta_{0}}\left(\frac{p_{0}}{p}\right)^{C_{v} / C_{p}}
$$

In Eq. (2), $C_{p}\left(=1004 \mathrm{~J} \mathrm{~K}^{-1} \mathrm{~kg}^{-1}\right)$ and $C_{v}\left(=717 \mathrm{~J} \mathrm{~K}^{-1} \mathrm{~kg}^{-1}\right)$ are the specific heats of air at constant pressure and volume respectively. $p_{0}$ is the base-state pressure, $\rho_{0}$ is the base-state density and $\theta_{0}$ is the base-state potential temperature. The horizontal velocities, atmospheric pressure, potential temperature and density are decomposed into initial time-invariant hydrostatic base-states $\left(u_{0}, v_{0}, p_{0}, \theta_{0}, \rho_{0}\right)$ which are a function of height only and the timevariant perturbations $\left(u^{\prime}, v^{\prime}, p^{\prime}, \theta^{\prime}, \rho^{\prime}\right)$ which are a function of both space and time:

$$
\begin{aligned}
& u(x, y, z, t)=u_{0}(z)+u^{\prime}(x, y, z, t) \\
& v(x, y, z, t)=v_{0}(z)+v^{\prime}(x, y, z, t) \\
& p(x, y, z, t)=p_{0}(z)+p^{\prime}(x, y, z, t) \\
& \theta(x, y, z, t)=\theta_{0}(z)+\theta^{\prime}(x, y, z, t) \\
& \rho(x, y, z, t)=\rho_{0}(z)+\rho^{\prime}(x, y, z, t)
\end{aligned}
$$

The prognostic equation for pressure deviation is approximated by:

$$
\frac{\partial p^{\prime}}{\partial t}+\frac{C_{p}}{C_{v}} p \frac{\partial u_{j}}{\partial x_{j}}=\rho_{0} g u_{j} \delta_{j 3}
$$

The conservation of energy is given in terms of potential temperature with the assumption of a dry adiabatic atmosphere:

$$
\frac{\partial \theta}{\partial t}=-\frac{\partial u_{j} \theta}{\partial x_{j}}+\theta \frac{\partial u_{j}}{\partial x_{j}}
$$

The system is closed by an equation of state for pressure:

$$
p=R_{d} \rho T
$$

In Eq. (10), $T$ is the air temperature and $R_{d}\left(=287 \mathrm{~J} \mathrm{~K}^{-1} \mathrm{~kg}^{-1}\right)$ is the gas constant for dry air. 


\section{Numerical Methods}

The numerical methods used in TASS are described in detail by Proctor (1987; Proctor 1996). A brief description is given here for the sake of completeness. The equations are discretized using a quadratic-conservative, fourth-order finite-differences in space for the calculation of momentum and pressure fields (Proctor 1996) and the third-order Leonard scheme (Leonard 1995) is used to calculate the transport of potential temperature. A Monotone Upstream-centered Scheme for Conservation Laws (MUSCL)-type scheme after van Leer (van Leer 1979; Ahmad and Proctor 2011) has also been implemented in TASS for the transport of scalar quantities. The TASS computational mesh uses the Arakawa-C grid staggering for specifying velocities and thermodynamic quantities (Arakawa and Lamb 1977). The Klemp-Wilhelmson time-splitting scheme (Klemp and Wilhelmson 1978) is used for computational efficiency in which, the higher-frequency terms given in the left hand side of Eq. (1) and (8) are integrated by enforcing the CFL criteria to take into account sound wave propagation due to compressibility effects. The remaining terms in Eq. (2) and (8) and Eq. (9) are integrated using a larger time step that is appropriate for anelastic and incompressible flows. Non-reflecting Orlanski boundary conditions (Orlanski 1976) are imposed at the outflow boundaries. A sixth-order filter is used to damp-out spurious oscillations that may arise due to the use of centered-differencing of momentum and pressure terms. The model code is written in FORTRAN 77 and has been fully parallelized for distributed computing platforms using the Message Passing Interface (MPI).

\section{Results}

In this section, the different benchmarks cases simulated to evaluate the hydrodynamic core of TASS are described in detail.

\section{A. Straka Density Current}

The non-linear density current benchmark (Straka et al. 1993) is presented in this section. The domain in this case was bounded from $-20 \mathrm{~km}$ to $20 \mathrm{~km}$ in the $x$-direction; from 0 to $5 \mathrm{~km}$ in the $y$-direction; and from 0 to $6.4 \mathrm{~km}$ in the $z$-direction. The mesh resolution was set to $50 \mathrm{~m}$ in both the $x$ - and $z$-directions and $1 \mathrm{~km}$ in $y$-direction. Outflow boundary conditions were used in the $x$-direction and periodic boundary conditions were used in the $y$-direction. The domain was initialized for a neutral atmosphere by setting the potential temperature at $300 \mathrm{~K}$. The initial $u$ velocity and the $w$-velocity were set to zero. The temperature profile was defined using the following relation:

$$
T(z)=300-\frac{z g}{c_{p}}
$$

A cold bubble was initialized by adding a perturbation in the temperature field using the following relation:

$$
\Delta T=\left\{\begin{array}{lc}
0.0 & \text { if } L>1.0 \\
-15.0\left[\frac{\cos (\pi L)+1.0}{2}\right] & \text { if } L \leq 1.0
\end{array}\right.
$$

where, $L=\sqrt{\left(x-x_{c} / x_{r}\right)^{2}+\left(z-z_{c} / z_{r}\right)^{2}}, x_{c}=0, z_{c}=3 \mathrm{~km}, x_{r}=4 \mathrm{~km}$ and $z_{r}=2 \mathrm{~km}$. As prescribed in Straka (1993), a constant eddy viscosity/diffusivity $\left(K_{m}=K_{h}=K=75 \mathrm{~m}^{2} / \mathrm{s}\right)$ was assumed for both the momentum and potential temperature fields. The simulation was run for time $=900 \mathrm{~s}$. The potential temperature perturbation $(\mathrm{K}), u$-velocity $(\mathrm{m} / \mathrm{s})$ and the $w$-velocity $(\mathrm{m} / \mathrm{s})$ fields at time $=900 \mathrm{~s}$ are shown in Figure 1. The figures show a portion of the actual computational domain from approximately 0 to $20 \mathrm{~km}$ in the $x$-direction and 0 to $6000 \mathrm{~m}$ in the $z$-direction. The simulation results shown in Figure 1 are in good agreement with the Straka benchmark, both qualitatively and quantitatively (see Table 1). In Table 1, REFC refers to the fully-compressible reference solution, REFS is the fully compressible reference solution on a staggered grid and REFQ is the reference solution using a quasi-compressible model. All reference solutions (REFC, REFS and REFQ) reported in Straka et al. (1993), were on grids with uniform resolution of $25 \mathrm{~m}$. f-waves refers to a Godunov-type scheme reported in Ahmad and Lindeman (2007). The data in Table 1 are for the right half of the computational domain (shown in Figure 1). The location of the front for the TASS solution is in terms of potential temperature and is given by the $x$-coordinate of the cell in the lowest level with a potential temperature of $299.99 \mathrm{~K}$. TASS solution also compares well with other reported simulations of the benchmark (e.g., see Janjic et al. 2001; Xue et al. 2000; Giraldo and Restelli 2008). 
Table 1: Comparison of the TASS results with the Straka Density Current Benchmarks

\begin{tabular}{|c|c|c|c|c|c|}
\hline Variable & $\begin{array}{c}\text { TASS } \\
\Delta x=\Delta z=50 \mathrm{~m}\end{array}$ & $\begin{array}{c}f \text {-waves } \\
\Delta x=\Delta z=50 \mathrm{~m}\end{array}$ & $\begin{array}{c}\text { REFC } \\
\Delta x=\Delta z=25 \mathrm{~m}\end{array}$ & $\begin{array}{c}\text { REFS } \\
\Delta x=\Delta z=25 \mathrm{~m}\end{array}$ & $\begin{array}{c}\text { REFQ } \\
\Delta x=\Delta z=25 \mathrm{~m}\end{array}$ \\
\hline$p_{\text {max }}^{\prime}(m b)$ & 1.31 & 1.26 & 2.87 & 2.49 & 1.74 \\
\hline$p_{\min }^{\prime}(\mathrm{mb})$ & -6.02 & -6.27 & -5.14 & -5.55 & -5.21 \\
\hline$\theta_{\text {max }}^{\prime}(\mathbf{K})$ & 0.0359 & $8.92 \mathrm{E}-03$ & 0.00 & 0.00 & 0.00 \\
\hline$\theta_{\min }^{\prime}(\mathbf{K})$ & -9.24 & -9.82 & -9.77 & -9.77 & -10.00 \\
\hline$u_{\max }(\mathrm{m} / \mathrm{s})$ & 37.35 & 34.44 & 36.46 & 35.02 & 34.72 \\
\hline$u_{\min }(\mathrm{m} / \mathrm{s})$ & -14.49 & -15.74 & -15.19 & -16.32 & -15.31 \\
\hline$w_{\max }(\mathbf{m} / \mathbf{s})$ & 12.17 & 13.62 & 12.93 & 13.28 & 13.04 \\
\hline$w_{\min }(\mathbf{m} / \mathbf{s})$ & -15.16 & -16.36 & -15.95 & -16.11 & -16.89 \\
\hline front location (m) & 15596.3 & 15525.00 & 15537.44 & $\mathrm{~N} / \mathrm{A}$ & 15509.17 \\
\hline
\end{tabular}
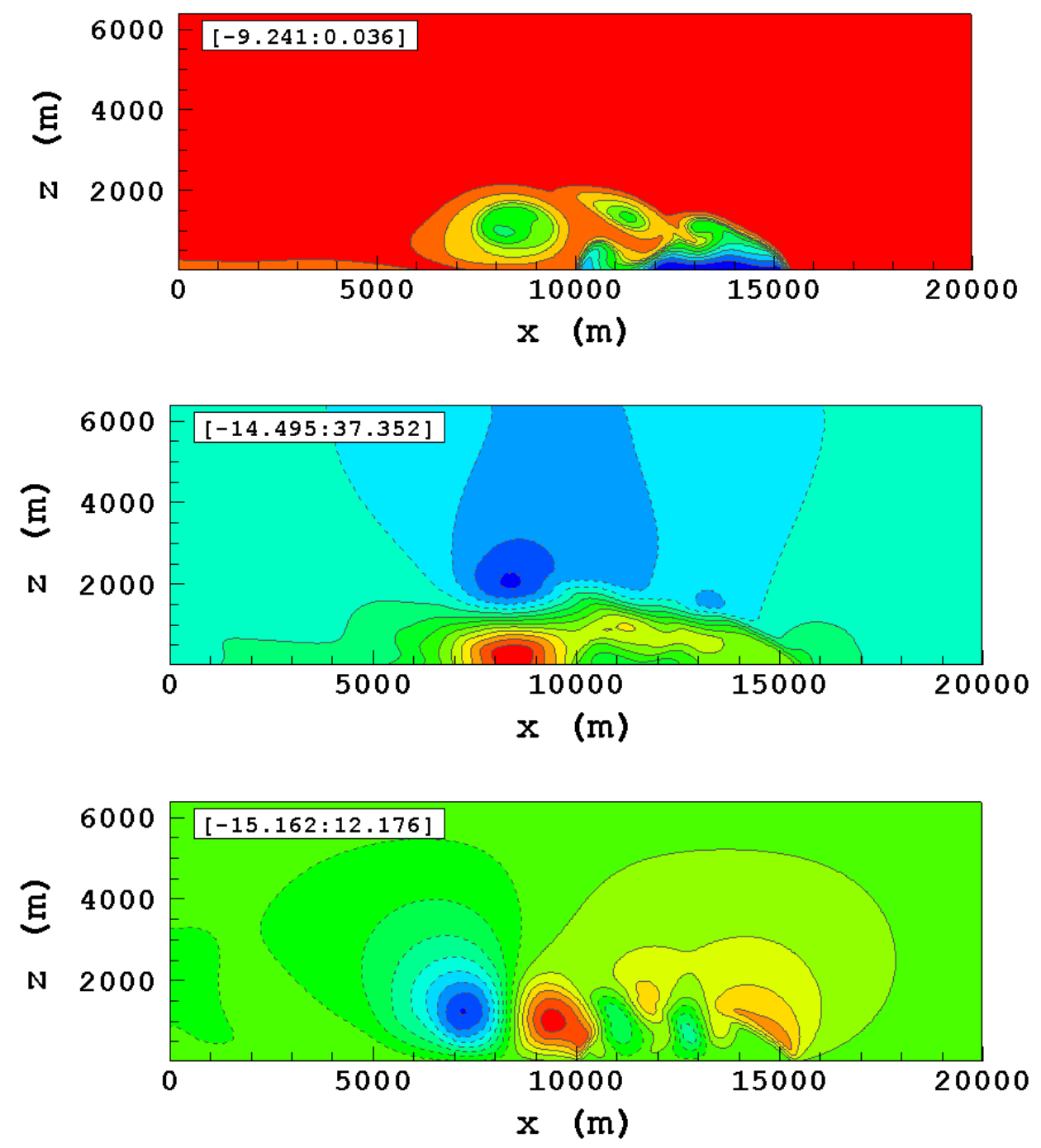

Figure 1. Non-Linear Density Current Benchmark. Potential temperature perturbation $(K)$ - top, $u$-velocity $(\mathrm{m} / \mathrm{s})-$ middle, and $w$-velocity $(\mathrm{m} / \mathrm{s})-$ bottom. Time $=900 \mathrm{~s} . \quad \Delta x=50 \mathrm{~m}$ and $\Delta z=50 \mathrm{~m}$. The maximum and minimum values of each field are given on the top-left corner of each figure. Dashed lines in the velocity graphics indicate negative values. Only the right half of the domain is shown in the figure. 


\section{B. Convection in Neutral Atmosphere}

There are many variants of this test case, e.g., see Koračin et al. (1998), Ahmad and Lindeman (2007) and Bryan and Fritsch (2002). TASS was evaluated using the setup similar to that described by Bryan and Fritsch (2002). The domain for this case was bounded from 0 to $20 \mathrm{~km}$ in the $x$-direction; from 0 to $5 \mathrm{~km}$ in the $y$-direction and from 0 to $10 \mathrm{~km}$ in the $z$-direction. The mesh resolution was set to $100 \mathrm{~m}$ in the $x$ - and $z$-directions and $1 \mathrm{~km}$ in the $y$-direction. Outflow boundary conditions were used in the $x$-direction and periodic boundary conditions were used in the $y$ direction. The top and bottom boundaries were set to solid walls (Bryan and Fritsch set wall conditions on the lateral boundaries as well). The domain was initialized for a neutral atmosphere at $\theta_{i}=300 \mathrm{~K}$ in hydrostatic balance. The initial $u$-velocity, $v$-velocity and the $w$-velocity were set to zero. A potential temperature perturbation in the form of a smooth cosine function was initialized near the lower boundary of the domain:

$$
\theta^{\prime}(x, z, t=0)=\Delta \theta_{0} \cos ^{2}\left(\frac{\pi L}{2}\right),
$$

where,

$$
L=\sqrt{\left(\frac{x-x_{c}}{x_{r}}\right)^{2}+\left(\frac{z-z_{c}}{z_{r}}\right)^{2}} .
$$

The potential temperature perturbation maximum $\Delta \theta_{0}$, was set to $2 \mathrm{~K}$. The center of the thermal perturbation was prescribed at $x_{\mathrm{c}}=10 \mathrm{~km}$ and $z_{\mathrm{c}}=2 \mathrm{~km}$. The radius of the perturbation was set to $2 \mathrm{~km}$ (i.e., $x_{\mathrm{r}}=z_{\mathrm{r}}=2 \mathrm{~km}$ ). The model was run for time $=1000 \mathrm{~s}$. The introduction of a warm potential temperature perturbation generates strong updrafts in the thermal core accompanied by downdrafts on either sides of the thermal. This is a highly non-linear case and as the thermal rises, large gradients in potential temperature develop at the top of the warm bubble. The TASS simulation results of potential temperature perturbation after time $=1000$ s is shown in Figure 2 along with results from the simulation by Bryan and Fritsch (2002). The TASS computed vertical velocity field after time $=1000 \mathrm{~s}$ is shown in Figure 3 along with results from the simulation by Bryan and Fritsch (2002). TASS was able to accurately simulate the convection in neutral atmosphere. The symmetry is well maintained in both the potential temperature and velocity fields and the results compare well qualitatively as well as quantitatively with the data reported by Bryan and Fritsch (2002). Bryan and Fritsch used a fifth-order upwind-biased scheme which may explain the slight differences in their solution.
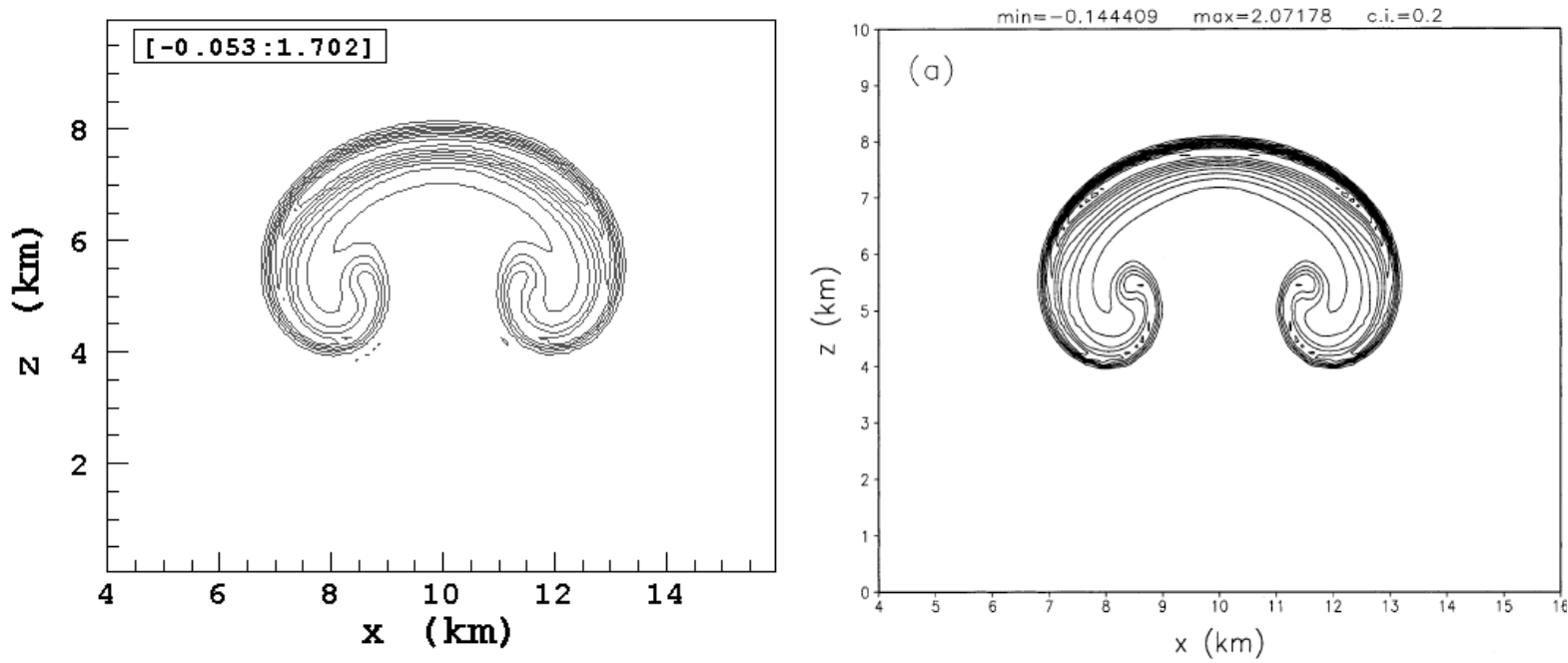

Figure 2. Convection in Neutral Atmosphere. Potential Temperature (K) field is shown in the figure. Comparison of the TASS computed solution (left) with the fifth-order upwind scheme (right) reported in Bryan and Fritsch (2002). Time $=1000 \mathrm{~s} . \Delta x=100 \mathrm{~m}$ and $\Delta z=100 \mathrm{~m}$. 

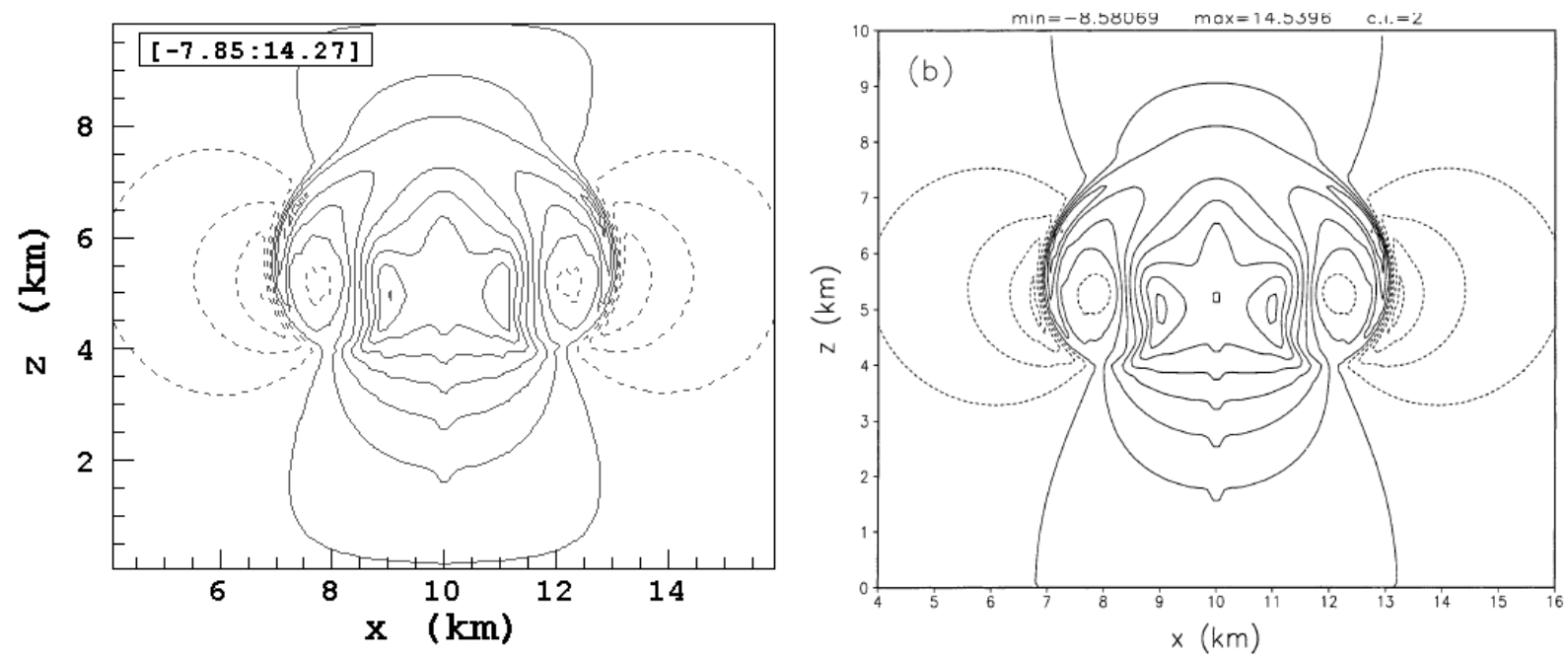

Figure 3. Convection in Neutral Atmosphere. Vertical velocity $(\mathrm{m} / \mathrm{s})$ field is shown in the figure. Comparison of the TASS computed solution (left) with the fifth-order upwind-biased scheme (right) reported in Bryan and Fritsch (2002). Time $=1000 \mathrm{~s}$. $\Delta x=100 \mathrm{~m}$ and $\Delta z=100 \mathrm{~m}$.

\section{Non-hydrostatic Inertia Gravity Waves}

The Skamarock-Klemp (1994) test for simulating inertia-gravity waves on the non-hydrostatic scale is described in this section. The domain was bounded from 0 to $300 \mathrm{~km}$ in the $x$-direction, from 0 to $5 \mathrm{~km}$ in the $y$-direction and from 0 to $10 \mathrm{~km}$ in the $z$-direction. Periodic boundary conditions were used in the lateral and the top and bottom boundaries were set to solid walls. The potential temperature in the vertical was initialized by a constant BruntVäisälä frequency $N=10^{-2} \mathrm{~s}^{-1}$. The waves were excited by an initial potential temperature perturbation given by:

$$
\theta(x, z, 0)=\Delta \theta_{0} \frac{\sin (\pi z / H)}{1+\left(x-x_{c}\right)^{2} / a^{2}}
$$

The amplitude of the initial potential temperature perturbation, $\Delta \theta_{0}$ was set to $10^{-2} \mathrm{~K}$. The height $H$ of the domain was $10 \mathrm{~km}$, the perturbation half width was $a=5 \mathrm{~km}$. The perturbation was initialized at $x_{c}=x_{\max } / 3$, where, $x_{\max }$ is the width of the domain $(300 \mathrm{~km})$. The initial $u$-velocity and $w$-velocity were set to zero. The model was run for time $=3000 \mathrm{~s}$. The mesh resolution in this simulation was set to $1 \mathrm{~km}$ in the horizontal and $100 \mathrm{~m}$ in the vertical. Due to the presence of gravity waves, the large time step used for advecting potential temperature in the timesplitting scheme was set to 2.5 times the acoustic time step.

Figure 4 shows the comparison of the centerline data at $z=z_{\max } / 2$ for $x=[0: 300 \mathrm{~km}]$ for TASS model, the WRF fifth-order upwind scheme and the WRF second-order centered scheme (see Ahmad and Lindeman 2007; Ahmad et al. 2007 for details on the WRF simulations). The TASS profile matches the WRF fifth-order solution quite well, whereas large dispersion and phase errors can be seen in the WRF second-order solution. The TASS solution on the other hand exhibits no noticeable diffusion, phase or dispersion errors. The potential temperature perturbation field for the TASS model after 3000s into the simulation is shown in Figure 5.

It should be noted that the solution in Skamarock and Klemp (1994) assumes a Boussinesq atmosphere and the computed results presented here are obtained from compressible flow models. The TASS results also compare well with other reported solutions of this benchmark (Ahmad et al. 2007; Ahmad and Lindeman 2007; Girlado and Restelli 2008). 


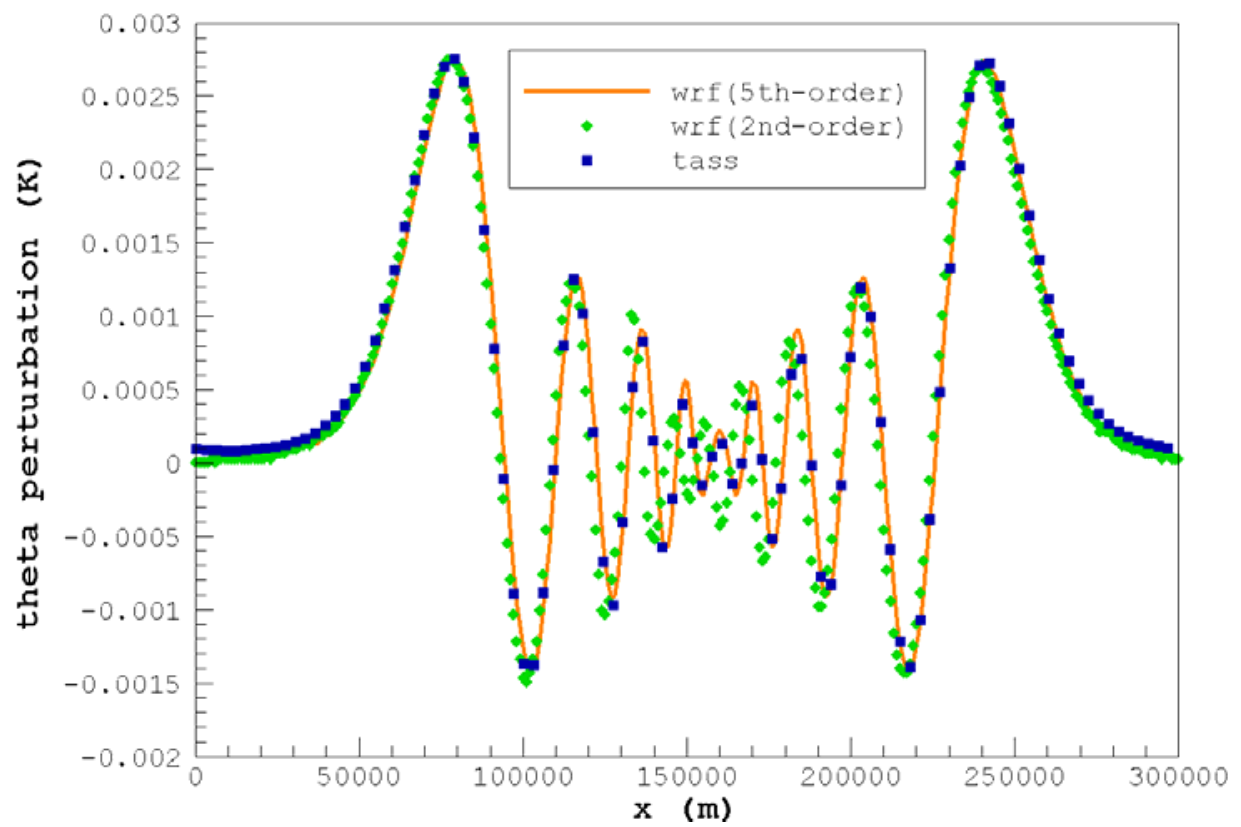

Figure 4. Non-hydrostatic Inertia-Gravity Waves. Comparison of the TASS model with the WRF fifth-order upwind scheme and the second-order centered scheme. Theta perturbation (K) values between $x=0$ \& $300 \mathrm{~km}$, for $\mathrm{z}=5 \mathrm{~km}$ are shown in the figure. $300 \times 100$ cells $(\Delta x=1 \mathrm{~km}$ and $\Delta z=100 \mathrm{~m})$. Time $=3000 \mathrm{~s}$.

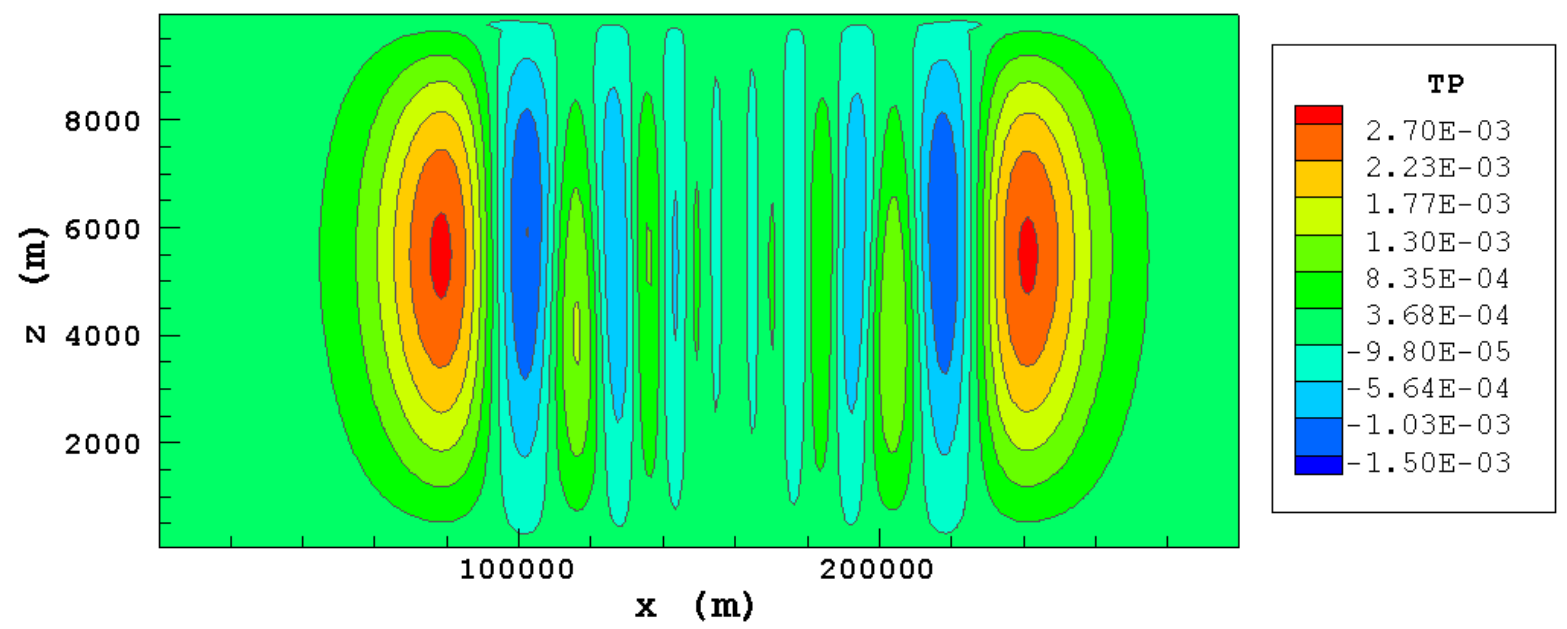

Figure 5. Non-hydrostatic Inertia-Gravity Waves. TASS computed potential temperature perturbation (K) field. Time $=3000 \mathrm{~s}$. 300x100 cells $(\Delta x=1 \mathrm{~km}$ and $\Delta z=100 \mathrm{~m})$. 


\section{Viscously Decaying Beltrami Flow}

A few closed-form solutions to the atmospheric flow equation sets have been found to exist under special circumstances. One such test case of the Beltrami flow was suggested by Shapiro (1993) for which an exact analytical solution exists. This test case is invaluable in verifying quantitatively, the accuracy and stability of the spatial and temporal discretization schemes implemented in an atmospheric flow model. The exact solution of the Beltrami flow is described in detail by Lilly (1986), Shapiro (1993), and Wang (1990). The simulations of the Beltrami flow using an earlier version of TASS are given in Switzer (1996). Please note that all of the previous cases presented in this paper described phenomena in two dimensions whereas the Beltrami test case is in three dimensions. The computational domain in this case comprised of $89 \times 59 \times 44$ cells and the mesh resolution was set to $3 \mathrm{~m}$ in the $x$-direction, $2 \mathrm{~m}$ in the $y$-direction and $1 \mathrm{~m}$ in the $z$-direction. The time-varying viscous Beltrami flow field is given by:

$$
\begin{gathered}
u(x, y, z, t)=-\frac{A}{k^{2}+l^{2}}[\lambda l \cos (k x) \sin (l y) \sin (m z)+m k \sin (k x) \cos (l y) \cos (m z)] \exp \left(-v \lambda^{2} t\right), \\
v(x, y, z, t)=\frac{A}{k^{2}+l^{2}}[\lambda k \sin (k x) \cos (l y) \sin (m z)-m l \cos (k x) \sin (l y) \cos (m z)] \exp \left(-v \lambda^{2} t\right), \\
w(x, y, z, t)=A \cos (k x) \cos (l y) \sin (m z) \exp \left(-v \lambda^{2} t\right), \\
p(x, y, z, t)=p_{s}-\rho\left[\frac{u^{2}+v^{2}+w^{2}}{2}+g z\right],
\end{gathered}
$$

where,

$$
\lambda^{2}=k^{2}+l^{2}+m^{2} .
$$

and,

$$
k=\frac{4 \pi}{L_{x}} ; \quad l=\frac{2 \pi}{L_{y}} ; \quad m=\frac{2 \pi}{L_{z}} .
$$

In Eq. (16)-(21), $k, l$ and $m$ are the wave numbers. $p_{\mathrm{s}}$ is the stagnation pressure at ground level and $A$ is the amplitude of the vertical velocity. The gradients of $u$-, $v$ - and $w$-velocity can be increased or decreased by changing the wave numbers $k, l$, and $m$. $v$ is the viscosity, $g$ is the acceleration due to gravity and $\rho$ is the fluid density. Initial conditions for the three components of the velocity field $(u, v$, and $w)$ and the pressure, $p$ can be obtained by setting the time, $t=0$. For the TASS simulations presented in this section, $A=2 \mathrm{~m} / \mathrm{s}, L_{\mathrm{x}}=267 \mathrm{~m}, L_{\mathrm{y}}=118 \mathrm{~m}$ and $L_{\mathrm{z}}=$ $44 \mathrm{~m}$ are the domain bounds in the $x-, y$ - and the $z$-direction respectively. Although, the exact Beltrami solution assumes an incompressible fluid, its use for validation of compressible flow models can be justified if the height of the computational domain is set such that it is much smaller than the density scale height (Shapiro 1993). The use of periodic boundary conditions in all directions is required for this test case. Two simulations were run with different values of viscosities $\left(v=1 \mathrm{~m}^{2} / \mathrm{s}\right.$ and $\left.v=0.1 \mathrm{~m}^{2} / \mathrm{s}\right)$. Periodic boundary conditions were used in all directions and the final integration time was set to $41 \mathrm{~s}$ as in Shapiro (1993).

Figure 6 shows the RMS error in domain maximum vertical velocity and Figure 7 shows the RMS error in domain total kinetic energy as a function of time. The errors for both the decaying and the weakly decaying cases are minimal. Figure 8 shows the computed $u-, v$-, and $w$-velocity fields in the $x-y$ plane at $z=10.5 \mathrm{~m}$. The difference between the exact and the computed solutions is shown in Figure 9. It can be seen that the errors in the TASS solution are very small and the fidelity of the solution is comparatively better than the Advanced Regional Prediction modeling System (ARPS) solution given in Shapiro (1993). Figure 10 shows the $u$-velocity and $w$ velocity values between $x=0$ and $267 \mathrm{~m}$, for $y=60 \mathrm{~m}$ and $z=10.5 \mathrm{~m}$. The computed TASS results are in excellent agreement with the exact solution. 


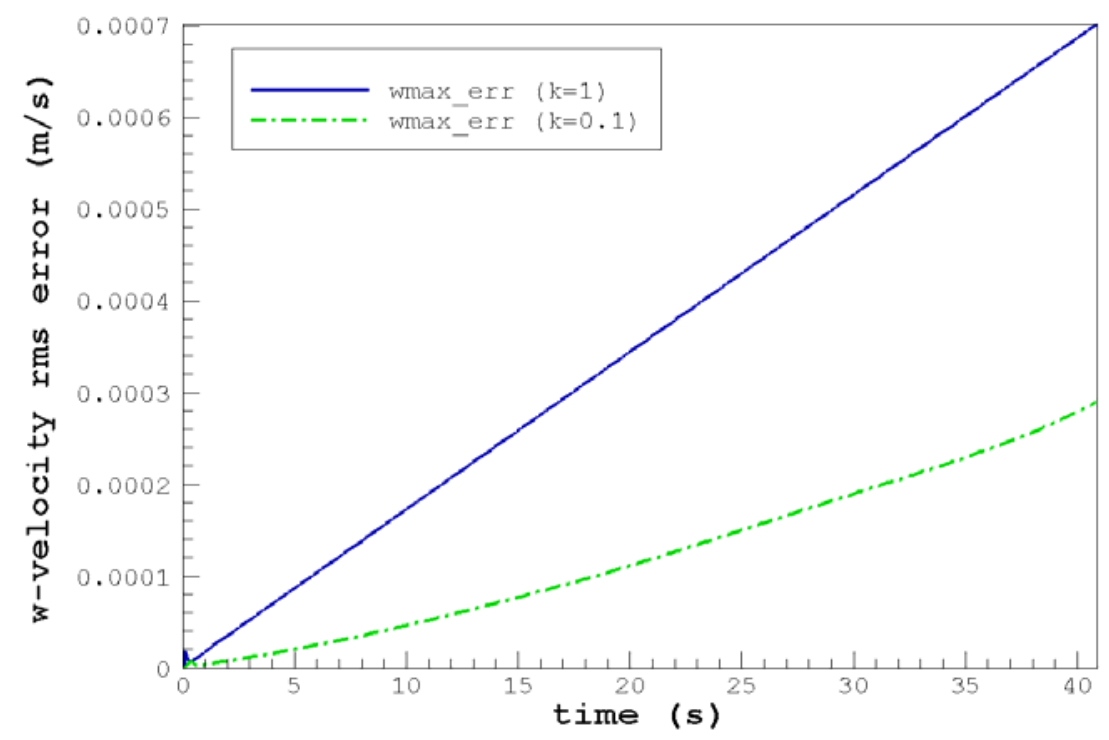

Figure 6. Viscously Decaying Beltrami Flow. Time history of RMS error in domain maximum w-velocity (top) and domain total kinetic energy (bottom) using different values of viscosities - the decaying case ( $v=$ $\left.1 \mathrm{~m}^{2} / \mathrm{s}\right)$ and the weakly-decaying case $\left(v=0.1 \mathrm{~m}^{2} / \mathrm{s}\right) .89 \times 59 \times 44$ cells. $\Delta x=3 \mathrm{~m}, \Delta y=2 \mathrm{~m}$, and $\Delta z=1 \mathrm{~m}$.

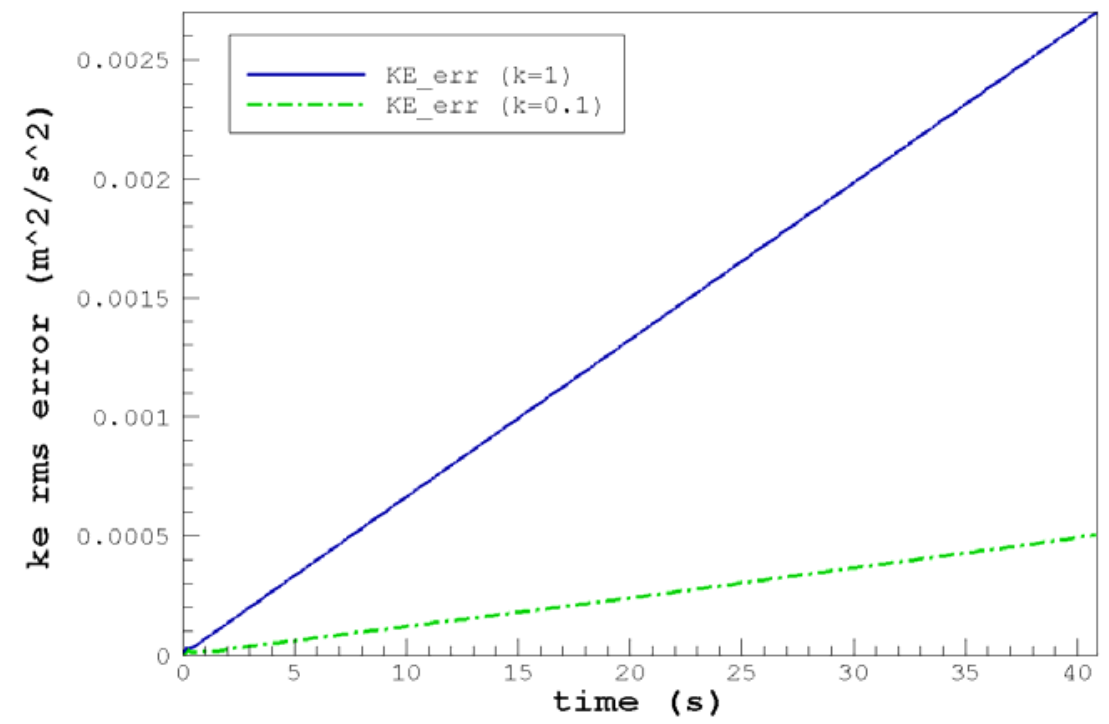

Figure 7. Viscously Decaying Beltrami Flow. Time history of RMS error in domain maximum $w$-velocity (top) and domain total kinetic energy (bottom) using different values of viscosities - the decaying case ( $v=$ $\left.1 \mathrm{~m}^{2} / \mathrm{s}\right)$ and the weakly-decaying case $\left(v=0.1 \mathrm{~m}^{2} / \mathrm{s}\right) .89 \times 59 \times 44$ cells. $\Delta x=3 \mathrm{~m}, \Delta y=2 \mathrm{~m}$, and $\Delta \mathrm{z}=1 \mathrm{~m}$. 


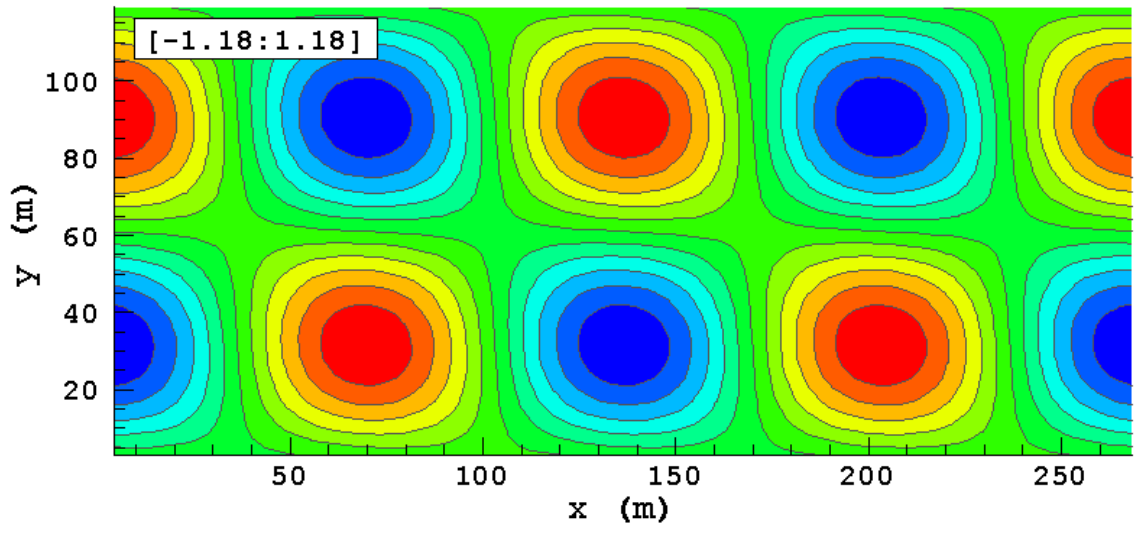

u-velocity $(\mathrm{m} / \mathrm{s})$

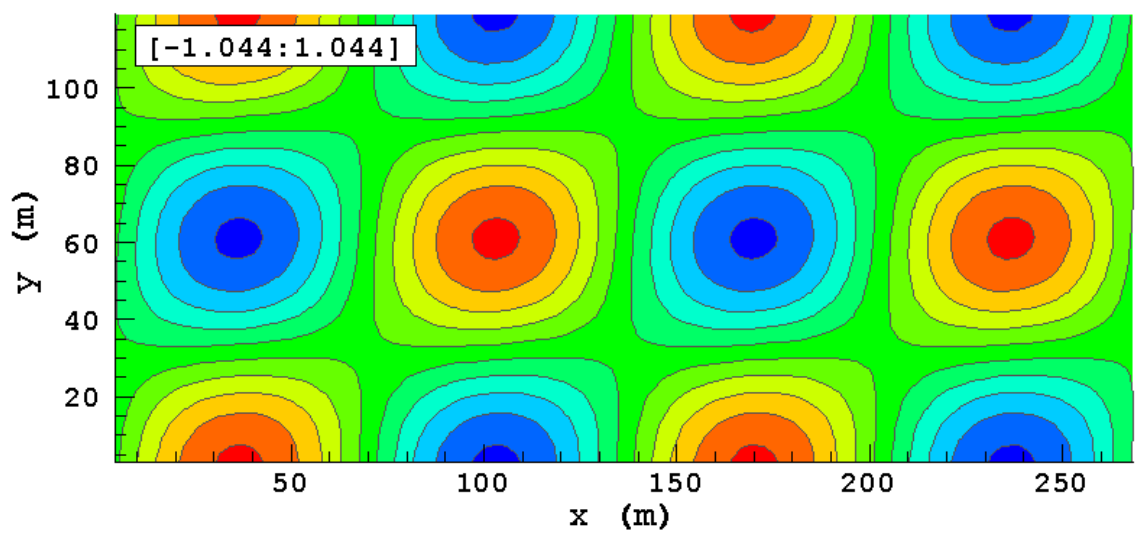

v-velocity $(\mathrm{m} / \mathrm{s})$

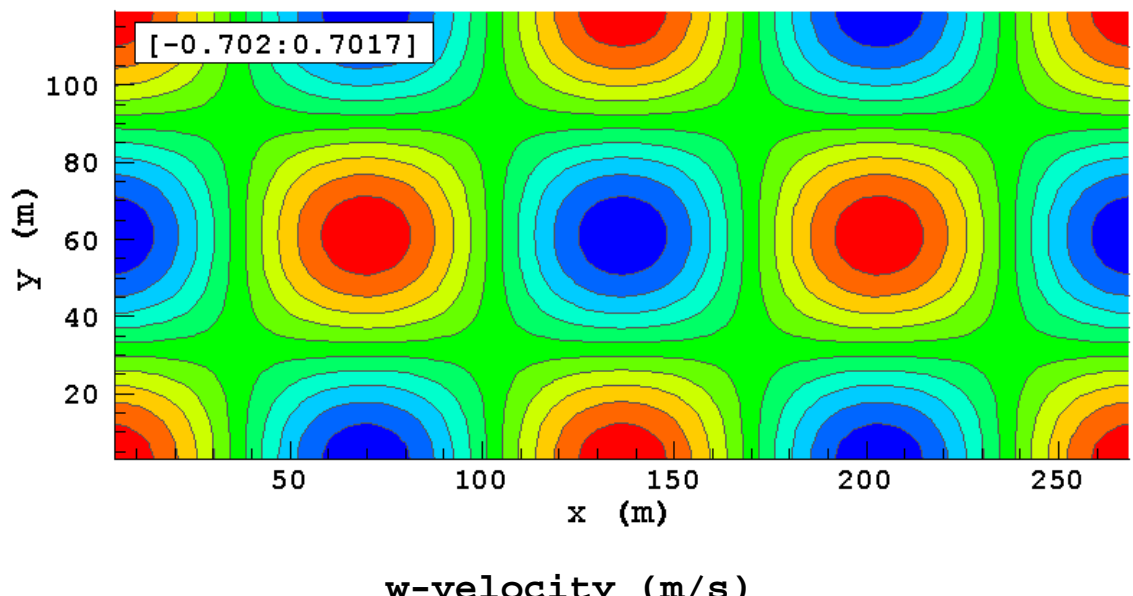

Figure 8. Viscously Decaying Beltrami Flow. TASS solution: $u$-velocity in $\mathbf{m} / \mathbf{s}$ (top panel); $v$-velocity in $\mathbf{m} / \mathbf{s}$ (middle panel) and the $w$-velocity in $\mathrm{m} / \mathrm{s}$ (bottom panel). The $x-y$ plane is shown at $z=10.5 \mathrm{~m}$. Viscosity coefficient, $v=1 \mathrm{~m}^{2} / \mathrm{s}$. Time $=41.02 \mathrm{~s}$. $89 \times 59 \times 44$ cells. $\Delta x=3 \mathrm{~m}, \Delta y=2 \mathrm{~m}$, and $\Delta z=1 \mathrm{~m}$. 

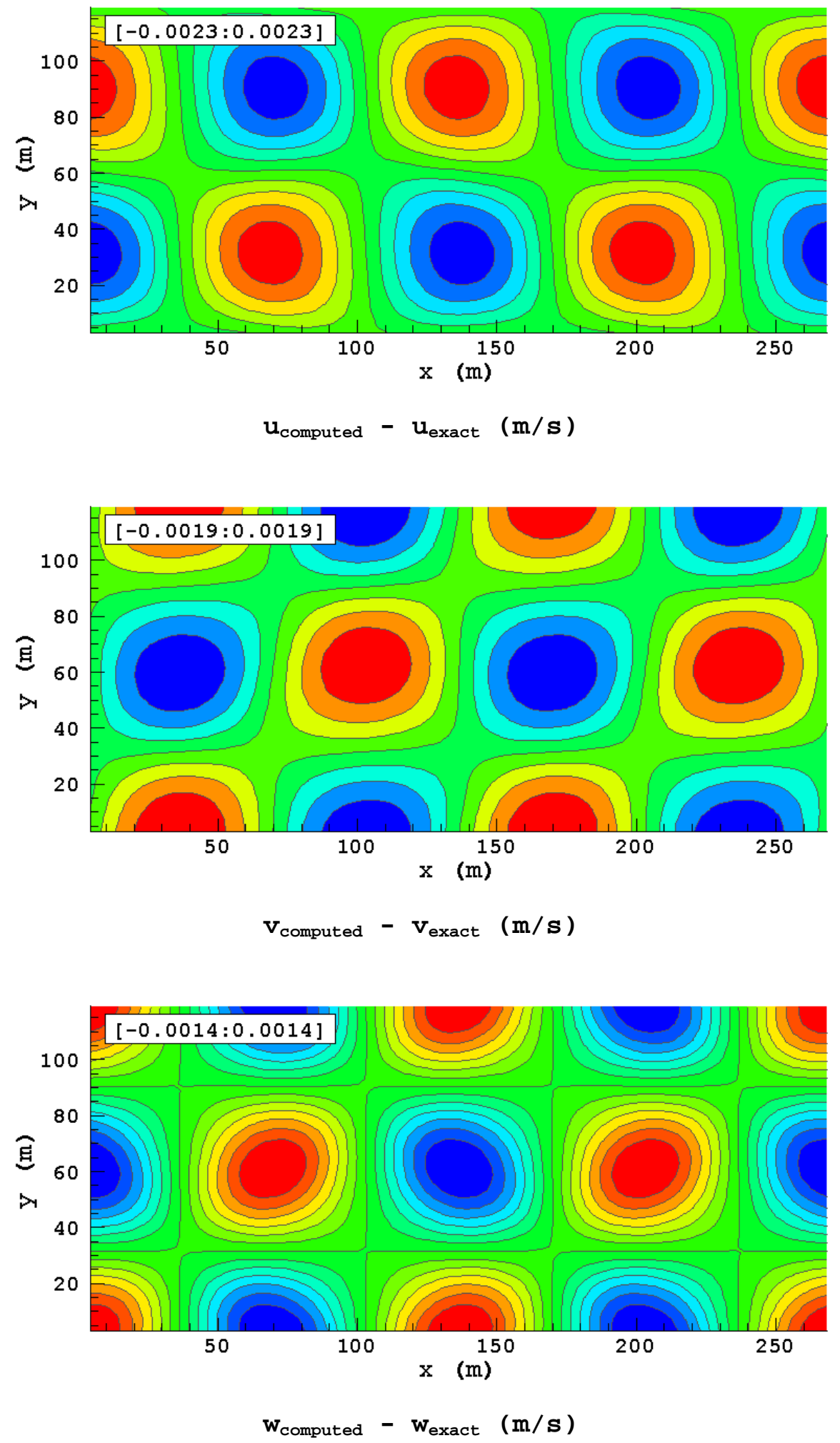

Figure 9. Viscously Decaying Beltrami Flow. Difference between computed and exact solutions: $u$-velocity in $\mathrm{m} / \mathrm{s}$ (top panel); $v$-velocity in $\mathrm{m} / \mathrm{s}$ (middle panel) and the $w$-velocity in $\mathrm{m} / \mathrm{s}$ (bottom panel). The $x$ - $y$ plane is shown at $z=10.5 \mathrm{~m}$. Viscosity coefficient, $v=1 \mathrm{~m}^{2} / \mathrm{s}$. Time $=41.02 \mathrm{~s}$. 89x59x44 cells. $\Delta x=3 \mathrm{~m}, \Delta y=2 \mathrm{~m}$, and $\Delta \mathrm{z}=\mathbf{1 m}$. 

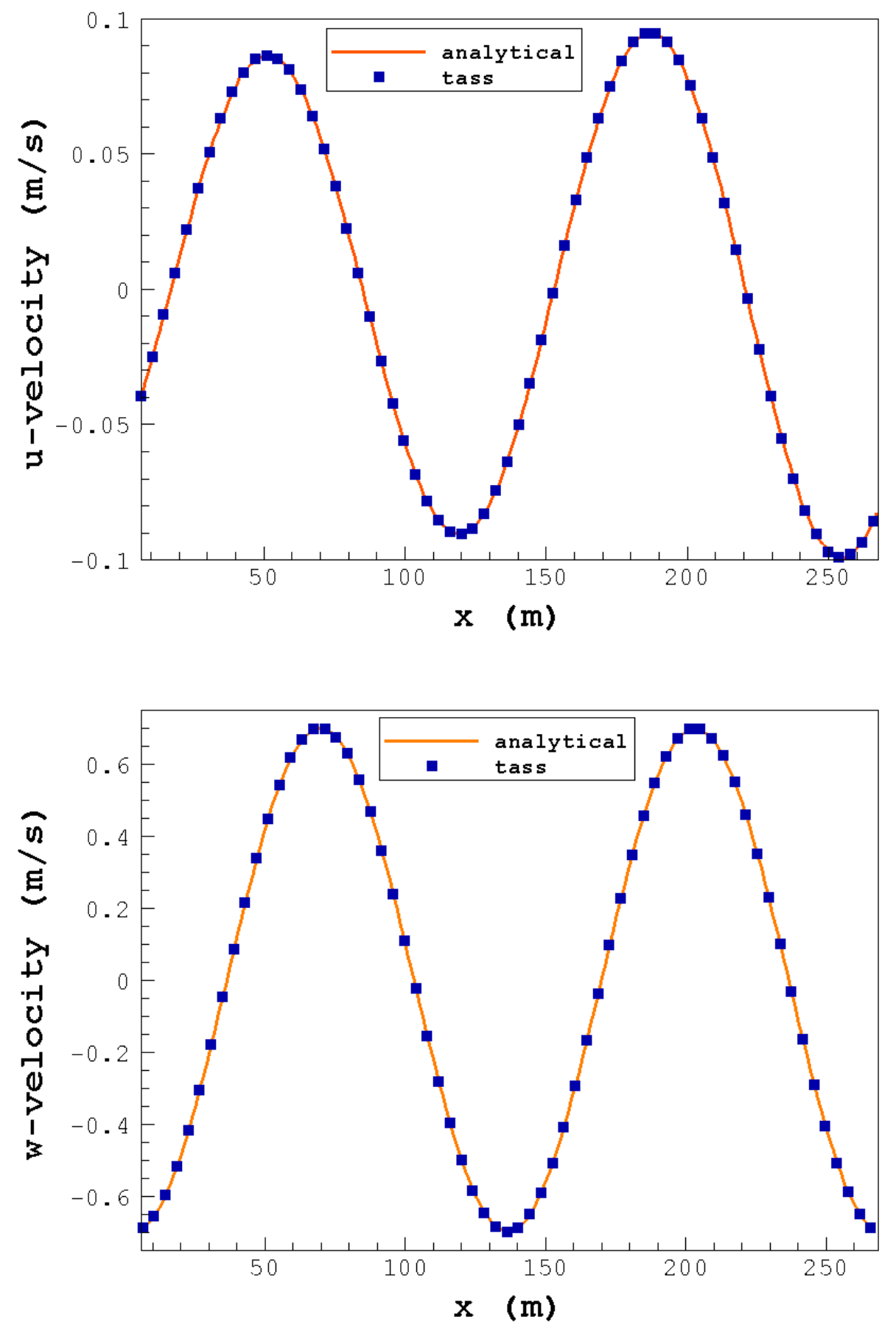

Figure 10. Viscously Decaying Beltrami Flow. Comparison of computed and exact solutions. $u$-velocity $(\mathrm{m} / \mathrm{s})$ values between $x=0 \& 267 \mathrm{~m}$, for $y=60 \mathrm{~m}$ and $z=10.5 \mathrm{~m}$ are shown in the top panel. $w$-velocity $(\mathrm{m} / \mathrm{s})$ values between $x=0 \& 267 \mathrm{~m}$, for $y=60 \mathrm{~m}$ and $\mathrm{z}=10.5 \mathrm{~m}$ are shown in the bottom panel. Viscosity coefficient, $v=$ $1 \mathrm{~m}^{2} / \mathrm{s}$. Time $=41.02 \mathrm{~s}$. $89 \times 59 \times 44$ cells. $\Delta x=3 \mathrm{~m}, \Delta y=2 \mathrm{~m}$, and $\Delta z=1 \mathrm{~m}$. 


\section{Summary}

The TASS hydrodynamics was evaluated against three benchmark cases in two dimensions and against an analytical test case in three dimensions with excellent results. The higher-order spatial and temporal discretization schemes implemented in TASS exhibit minimal dispersion, phase and diffusion errors. The simulation results also compare well with other models. In the inertia-gravity wave case, for example, the TASS model performs at par with the fifth-order upwind biased scheme implemented in WRF and out-performs WRF's second-order centered scheme which exhibits large dispersion and phase errors. In the case of Beltrami flow, again the TASS model performed exceptionally. In terms of accuracy, TASS also out-performs the ARPS model for Beltrami simulation (see Shapiro 1993). Due to its higher order spatial accuracy and minimal dispersion and phase errors, TASS is well suited for simulating complex turbulent flows and convection dominated flows.

\section{Acknowledgments}

This work is sponsored under NASA's Concepts \& Technology Development Project of the Airspace Systems Program. The numerical simulations were conducted using the NASA Pleiades supercomputer cluster.

\section{References}

${ }^{1}$ Ahmad, N., J. Lindeman, “Euler Solutions using Flux-based Wave Decomposition”. International Journal for Numerical Methods in Fluids, Vol. 54:1, 2007, pp. 41-72.

${ }^{2}$ Ahmad, N., D. Bacon, A. Sarma, D. Koracin, R. Vellore, Z. Boybeyi, and J. Lindeman, "Simulations of Non-hydrostatic Atmosphere using Conservation Laws Package”, AIAA Paper 2007-84.

${ }^{3}$ Ahmad, N., and F. Proctor, "Advection of Microphysical Scalars in Terminal Area Simulation System (TASS)", Accepted for presentation at the $49^{\text {th }}$ AIAA Aerospace Sciences Meeting, Orlando, Florida, January 2011.

${ }^{4}$ Arakawa, A., V. R. Lamb, "Computational design of the basic dynamical process of the UCLA general circulation model", Methods in Computational Physics, Vol. 17, 1977, pp. 173-265.

${ }^{5}$ Bacon, D. P., and R. A. Sarma, "Agglomeration of dust in convective clouds initialized by nuclear bursts", Atmospheric Environment, Vol. 25, 1991, pp. 2627-2642.

${ }^{6}$ Bacon, D. P., R. A. Sarma, F. H. Proctor, "Smoke injection into the atmosphere from large area fires", Defense Nuclear Agency Technical Report DASIAC-TN-87-35-V1. 1986.

${ }^{7}$ Bryan, H. B., and M. Fritsch, "A Benchmark Simulation for Moist Nonhydrostatic Numerical Models", Monthly Weather Review, Vol. 130, 2002, pp. 2917-2928.

${ }^{8}$ Giraldo, F. X., and M. Restelli, “A study of spectral element and discontinuous Galerkin methods for the Navier-Stokes equations in nonhydrostatic mesoscale atmospheric modeling: Equations sets and test cases", Journal of Computational Physics, Vol. 227, 2008, pp. 3849-3877.

${ }^{9}$ Han, J., Lin, Y. -L., Schowalter, D. G., Arya, S. P., and Proctor, F. H., "Large Eddy Simulation of Aircraft Wake Vortices within Homogeneous Turbulence: Crow Instability," AIAA Journal, Vol. 38, 2000a, pp. 292-300.

${ }^{10}$ Han, J., Lin, Y.-L., Arya, S.P., and Proctor, F.H., "Numerical Study of Wake Vortex Decay and Descent within Homogeneous Turbulence," AIAA Journal, Vol. 38, 2000b, pp. 643-656.

${ }^{11}$ Janjic, Z. I., J. P. Gerrity, and S. Nickovic, "An Alternative Approach to Nonhydrostatic Modeling", Monthly Weather Review, Vol. 129, 2001, pp. 1164-1178.

${ }^{12}$ Klemp, J. B., and R. Wilhelmson, "The simulation of three-dimensional convective storm dynamics", Journal of Atmospheric Sciences, Vol. 35, 1978, pp. 1070-1096.

${ }^{13}$ Klemp, J. B., W. C. Skamarock, and J. Dudhia, "Conservative Split-Explicit Time Integration Methods for the Compressible Nonhydrostatic Equations", WRF Eulerian Prototype Model Equations Draft Manuscript, National Center for Atmospheric Research, 2000. 
${ }^{14}$ Koračin, D., V. Isakov and L. Mendez-Nuñez, “A cloud-resolving model with the radiation scheme based on the Monte Carlo method", Atmospheric Research, Vol. 47, 1998, pp. 437-459.

${ }^{15}$ Leonard, B. P., M. K. MacVean and A. P. Lock, "The Flux-Integral Method for Multidimensional Convection and Diffusion”, Applied Mathematical Modeling, Vol. 19, 1995, pp. 333-342.

${ }^{16}$ Lilly, D., "The structure, energetic and propagation of rotating convective storms. Part II: Helicity and storm stabilization", Journal of Atmospheric Science, Vol. 43, 1986, pp. 126-140.

${ }^{17}$ Orlanski, I., “A simple boundary condition for unbounded hyperbolic flows”, Journal of Computational Physics, Vol. 21. 1976, pp. 251-269.

${ }^{18}$ Proctor, F. H., “The Terminal Area Simulation System / Volume 1: Theoretical Formulation”, NASA Technical Report 4046. 1987.

${ }^{19}$ Proctor, F. H., “The Terminal Area Simulation System / Volume 2: Verification Cases”, NASA Technical Report 4047. 1987.

${ }^{20}$ Proctor, F. H., "Numerical Simulation of Wake Vortices Measured During the Idaho Falls and Memphis Field Programs", AIAA Paper 1996-2496.

${ }^{21}$ Proctor, F. H., D. W. Hamilton, and R. L. Bowles, "Numerical Study of a Convective Turbulence Encounter", AIAA Paper 2002-0944.

${ }^{22}$ Proctor, F. H., "Interaction of Aircraft Wakes from Laterally Spaced Aircraft", AIAA Paper 2009-343.

${ }^{23}$ Proctor, F. H., and R. L. Bowles, "Three-Dimensional Simulation of the Denver 11 July 1988 Microburst-Producing Storm", Meteorology and Atmospheric Physics, Vol. 49, 1992, pp. 107-124.

${ }^{24}$ Shapiro, A., "The Use of an Exact Solution of the Navier-Stokes Equations in a Validation Test of a Three-Dimensional Nonydrostatic Numerical Model”, Monthly Weather Review, Vol. 121, 1993, pp. 2420-2425.

${ }^{25}$ Skamarock, W. C., and J. B. Klemp, "Efficiency and Accuracy of the Klemp-Wilhelmson Time-Splitting Technique", Monthly Weather Review, Vol. 122, 1994, pp. 2623-2630.

${ }^{26}$ Smagorinsky, J., “General Circulation Experiments With the Primitive Equations", Monthly Weather Review, Vol. 91, 1963, pp. 99-164.

${ }^{27}$ Straka, J. M., R. B. Wilhelmson, L. J. Wicker, J. R. Anderson, and K. K. Droegemeier, "Numerical Solutions of a Nonlinear Density Current: A Benchmark Solution and Comparisons”, International Journal for Numerical Methods in Fluids, Vol. 17, 1993, pp. 1-22.

${ }^{28}$ Switzer, G., "Validation Tests of TASS for Application to 3-D Vortex Simulations", NASA Technical Report 4756.1996.

${ }^{29}$ Stull, R., “An Introduction to Boundary-Layer Meteorology”, Kluwer Academic Publishers. 1997.

${ }^{30}$ van Leer, B., 1979: Towards the Ultimate Conservative Difference Scheme. V. A Second-Order Sequel to Godunov's Method, Journal of Computational Physics, Vol. 32, 1979, pp. 101-136.

${ }^{31}$ Wang, C., Y., "Exact solutions of the Navier-Stokes equations - the generalized Beltrami flows, review and extension". Acta Mechanica, Vol. 81, 1990, pp. 69-74.

${ }^{32}$ Wicker, L. J., and W. C. Skamarock, "A Time-Splitting Scheme for the Elastic Equations Incorporating Second-Order Runge-Kutta Time Differencing”, Monthly Weather Review, Vol. 126, 1998, pp. 1992-1999.

${ }^{33}$ Xue, M., K. K. Droegemeier, and V. Wong, "The Advanced Regional Prediction System (ARPS) - A multi-scale nonhydrostatic atmospheric simulation and prediction model. Part I: Model dynamics and verification", Meteorology and Atmospheric Physics, Vol. 75, 2000, pp. 161-193. 